\title{
Molecular Dynamics: A Tool for Undergraduate Engineering Students to Transform Their Understanding of Chemistry at Molecular Level
}

\author{
Meena B. Singh ${ }^{1}$, Vilas G. Gaikar ${ }^{2}$ \\ ${ }^{1,2}$ Department of Chemical Engineering, Institute of Chemical Technology, Matunga, Mumbai - 400019 , India \\ ${ }^{1}$ meena18s@yahoo.co.in \\ ${ }^{2}$ v.g.gaikar@gmail.com
}

\begin{abstract}
Molecular dynamics is a valuable tool in the field of chemistry that can be introduced as part of curriculum for engineering students at undergraduate (UG) level for better understanding of chemistry. Particularly engineering UG students have difficulty in understanding chemistry in terms of the molecular structures. Newtonian mechanics on the other hand is easily understood by the students pursuing engineering. Molecular dynamics, the branch of computational chemistry therefore can help these students to understand chemistry better if students also interpret the data and images produced by MD simulation. This case study describes the use of GROMACS, an open source code and visualizing packages like CHIMERA and VMD as a tool to perform MD simulations, which successfully reformed the approach of undergraduate students towards chemistry. The Molecular dynamics workshop was conducted for undergraduate students during summer vacation of 2015 to emphasize on conceptual understanding of chemistry and physics, using real life examples of mass transfer of compounds from one phase to another phase. This exercise encouraged the participating students to initiate their own learning in the field of molecular modeling.
\end{abstract}

Keywords: Molecular dynamics simulation; GROMACS; chemistry, Undergraduate students; Workshop; Separation and extraction

\footnotetext{
Meena B. Singh ${ }^{1}$

${ }^{1,2}$ Department of Chemical Engineering, Institute of Chemical Technology, Matunga, Mumbai - 400 019, India

${ }^{1}$ meena18s@yahoo.co.in
}

\section{Introduction}

Chemistry is an integral part of engineering undergraduate curriculum. Usually the subject is taught in the class and few experimental studies in laboratory. But for most students the visualization of molecules, particularly their existence in 3D world, is difficult as the chemicals are usually shown on a plane in 2D format. The lack of visualization affects not only the learning but also interest in the chemistry itself. The various theories of chemical bonding and structure like Hückel MO theory, Lewis structure theory, VSEPR theory, etc. are attempts to predict the chemical nature and properties which many students fail to understand.

On the other hand, advances in theoretical chemistry and availability of increasing computational powers at cheaper rates allow the chemists to explore the field without conducting large number of experiments. Numerous packages, paid and free, for molecular simulation are available on the net and with appropriate visualization softwares can enrich the learning experience. The molecular modeling first defines or estimates the characteristic of the material and then performs the simulation based on laws of physics. The question we ask is whether we can use molecular modeling in the classroom to train the students better in the foundations of chemistry by engaging them in an enjoyable manner. The various models of molecular modeling can help students to comprehend chemistry by "viewing" molecules and "sensing" their surroundings in any physical or chemical processes. Introduction and demonstrate of various molecular simulation techniques in the classroom are reported in various articles. (Paselk and Zoellner, 2002, Whisnant et al., 2000, Taylor and Feller, 2002, Cramer et al., 2001)

We propose here use of basic student friendly molecular modeling tools in classroom teachings and laboratory practices. Engineering students understand the Newtonian Mechanics very well and, therefore, Molecular Dynamics (MD) is an apt part of molecular modelling approach in getting the students involved in their own learning by doing 
the simulations. The MD calculations are based on estimating the net force working on each molecule of the system from the gradient of the potential field and then corresponding displacement and motion, using the Newton's Second law of motion. Familiarity with the basic laws of physics makes the engineering students to grasp the concept better. Molecular simulations also bring the innovation in the method of synthesis of compounds and their characterization.

In this paper, we describe our attempt to help the undergraduate engineering students in better understanding of chemistry through a molecular dynamics simulation workshop.

\section{Methods}

Simulation engine used here was GROMACS 5.0(Berendsen et al, 1995, Lindahl et al., 2001, Berendsen et al., 1984) which is an open source code for performing MD simulation. GROMACS simulates a Newton 2nd law of motion for various systems ranging from hundreds to many millions of atoms. GROMACS can handle biological systems like lipids, proteins and amino acids, but since GROMACS can also calculate non-bonded interactions very fast, it is also widely used for non-biological systems like polymers, liquid-liquid extraction, metal ion extraction etc. This program is very user-friendly, where all parameters and topology files are written in ' $\mathrm{C}$ ' language. It contains many fail safes inbuilt in the program, if anything goes wrong during simulation clear error warning gets displayed on the command prompt. During simulation, GROMACS also notifies to the user the number of steps completed out of the total number of expected steps and when the simulation is likely to be over. There are also many inbuilt tools in GROMACS for analysis of trajectories and physiochemical properties, so there is no need to write separate codes for analysis of the data generated by MD simulation. CHIMERA (Pettersen et al., 2004) and VMD (Humphrey et al., 1996) were used in this workshop as basic visualizer as they are easy to operate.

\section{Procedure}

Considering that the training in molecular simulation is so distinctive from the conventional classroom room practices, a one month intensive workshop was conducted on molecular dynamics (MD) for 1st year undergraduates. This was an innovative approach to teach chemistry and various molecular processes to the engineering and technology students. The workshop was conducted in the Information Processing Centre of Institute of Chemical Technology (Mumbai) during the summer vacation of 2015. The choice to participate in the workshop was given immediately after the semester examination. Only those students, who were interested in learning something new, therefore, participated. A total 30 students from various departments of the University like Chemical Engineering, Food Engineering and Technology, Dyestuff Technology, Pharmaceutical Sciences and Technology and Polymer and Surface Engineering participated in the activity. Some of the students were from other Engineering Institute. All these students had basic working knowledge of chemistry; therefore, they were perfect candidates for the workshop.

The aim of the workshop was to encourage students to utilize molecular dynamics (MD) as a tool for self-learning chemistry by exploring various physical processes that occur at the molecular levels. Students were also encouraged to probe into various input parameters for the same system and their effects on physical and structural properties of the system. During the 1st week of the workshop, the students learnt the installation of open source code, GROMACS and later went through a common tutorial with hands-on-experience on basics of molecular dynamics. GROMACS helps in studying the interaction of compounds in the solutions as a function of temperature and pressure. The utilities built-in the code aid in the calculation of local structures as radial distribution function and macroscopic properties such as diffusivity coefficients, densities, viscosity and potential energies of the systems. GROMACS simulates long range interactions in the liquid state, which appears due to distinct molecular interactions. Short range interactions are also very important to study as they give the idea about the coordination structures of a given species in the solution.

Initially students struggled while drawing structures of the compound and then listing the each atom name in input file with the respective partial charges on of every atom. The main points was not to miss any atom while writing the input file and then assign those correct charges, so that when they run the program, they could get overall charge neutral on the molecule and not any atom appear in unbounded form. Since the molecules of interest were nonbiological in origin, participants needed to search for the forcefield parameters in various research publications and also needed to specify the bond length and bond angles between the atoms. This whole task of writing an initial input file was very lengthy and exhaustive process for the students. Any minor mistake would result in the error message, which got displayed on the computer screen e.g. system with non- zero charge, bonds, bond angles and dihedrals not found etc. few of the students thought that they can't follow this lengthy and tiresome process of writing an input file and they left after 3-4 days, but at the same time they also realized that learning and gaining knowledge from this activity is tremendous, so they eventually rejoined the program.

At the end of the first week, separate small projects were given to each of the students, which they could work on their own for next three weeks. These projects were linked so that each one would compare the results with others as well work towards a bigger project where they could see the direct implementation of their simulation results, in addition to estimation of some physical properties of the compounds which could be compared to the data in literature for validation.

The system considered for the workshop was mostly liquid-liquid extraction. Liquids are specifically very alluring to study by MD simulations because, even though they don't display macroscopic arrangement of solids, they tend to acquire distinct arrangement at molecular level (Larry Dukerich, 2015). This liquid-liquid extraction 
studies involve extraction of various aromatic compounds and metal ions from one phase to another phase either in the presence or absence of ligand. Basically, one project is divided between 2-4 students and each of them worked independently and then compared their data with each other to complete the project.

\section{Impact on Learning}

Most of students from different branches of science and technology portray the workshop experience as having a completely different perspective towards the subject chemistry. The students can now draw and visualize the 3D structures of a chemical compound by using various visualization tools like CHIMERA and VMD. Being able to draw and see the three dimensional structure of species enables the students to visualize the size of molecules, and phenomena like steric crowding and steric hindrance due to the presence of side chains in the molecules. They are also able to appreciate localized charges on different atoms and various inter- and intra-molecular forces of interaction. The charge distribution on the molecules provides the knowledge about the behavior of molecules in various solvents. These candidates familiarized themselves with many LINUX based software which are generally used in molecular dynamic simulations.

They have also learnt about the selection of force-field parameters from various research papers and their used for the MD simulation. In the process, they also learnt how to analyzing the research paper and carryout target oriented literature survey, which is an important accomplishment for students at an undergraduate level. Students were very fascinated by seeing how the input force field parameters affect the result of MD simulations. Many students were simply delighted to observe how varying the temperature affects the various physiochemical properties like viscosity, diffusion coefficient, density etc. of liquid. They have also grasped the knowledge about how to link the various macroscopic properties of different liquids to their molecular structure. This workshop also enabled them to understand about short-range interactions and long range interactive forces. They also learnt about the cut-off distances and periodic boundary conditions of the

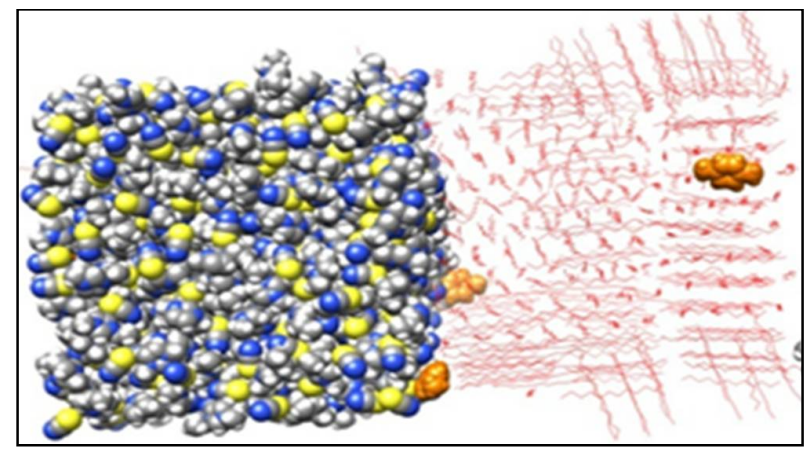

Figure 01. Extraction of aromatic hydrocarbon. The molecule represented by orange sphere model is the principle compound which is getting extracted from dodecane phase (represented by red lines model) into the extractant (represented by left side of image i.e the combination of blueyellow and grey sphere model) simulation box. Molecular dynamics is a real-time and interactive study, which enabled the students to visualize various chemical reactions at the molecular level, and to establish a relationship between chemical reaction equations and various molecular interactions. The participants seem to learn better by observing chemical reactions and extraction of compounds as a time dependent process. Students now can construct perceptive and comprehensive model of various atomic interactions in their mind so that they can comprehend the chemical reactions (Melanie et al., 2015, Pallant and Tinker, 2004).

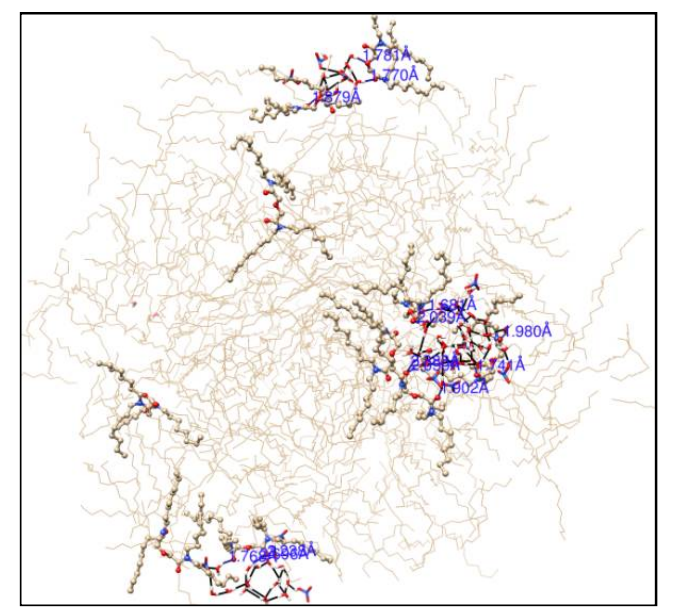

Figure 02. Cluster formed by the extractant (ligand) in the organic phase. The molecules represented by stick model are the ligands which are forming cluster with each other and with some water molecules and nitric acid molecules (represented by stick model) in the dodecane phase (represented by grey lines in the background)

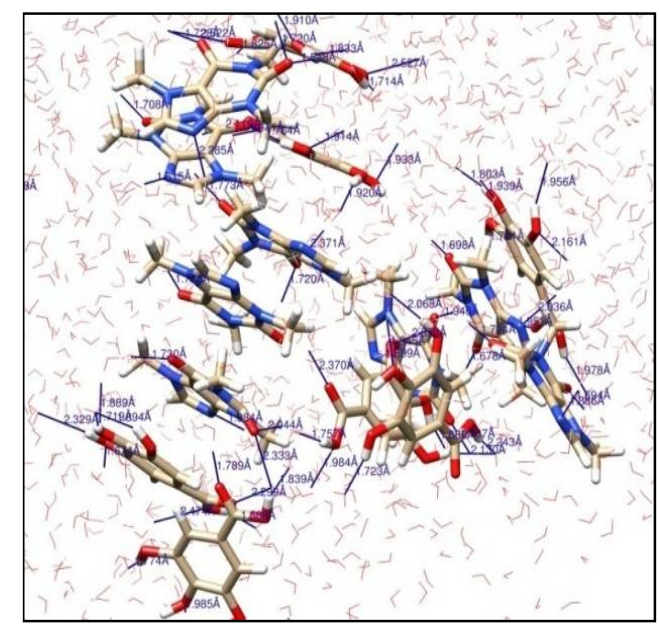

Figure 03. Pi-Pi stacking formed by the tea components in the water. The molecules represented by stick model are the various tea components which posses various aromatic groups are exhibiting pi-pi stacking in aqueous phase i.e water (represented by grey lines in the background) 


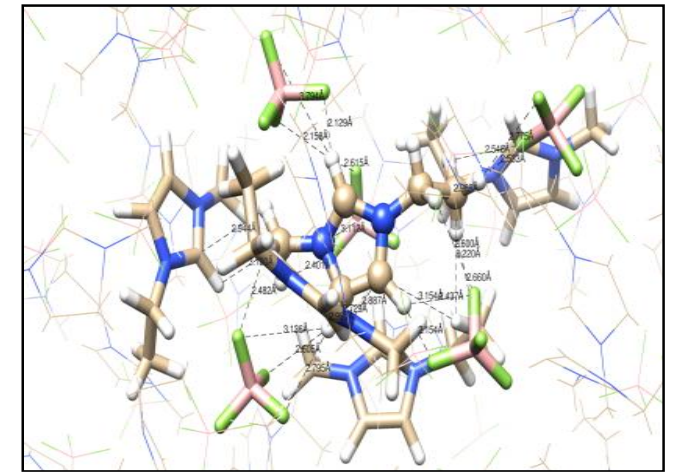

Figure 04. Electrostatic interaction between imidazolium cation and tetrafluroborate anion. The molecules represented by blue-grey ball and stick model and stick model is the imidazolium cation which is interacting with surrounding tertrafluroborate anions (represented by green-pink stick model)

Without proper analysis of any simulation there is no meaning of doing it. These students have learned how to analyze the simulation data by calculating various properties like densities of the solution, the diffusion coefficient of various species in the solution, viscosity, radial distribution functions and total energy of a system which gives them idea about the stability of the systems.

They have also done structural analysis of the systems to determine the first and second coordination shells of the particular species, which facilitates understanding the coordination structures and the coordination number of the species in the solvated conditions. For better understanding of extraction process, students have also converted their MD trajectory files into the movie with the aid of VMD software, in which they can actually visualize the movement of molecules with respect to time and see how the molecule of interest is getting extracted as shown in Figure 01. They have also analyzed various molecular clusters formed during the simulation in the box as shown in Figure 02. Molecular clusters are the aggregation of the same or different molecules at a specific site in the simulation box. Molecular clusters have been previously studied for various organic and inorganic systems by performing MD simulations (Sabelli, 1994, Flöck et al., 2006, Singh et al., 2015). The students have also become very observant and they could easily spot the intermolecular interactions, e.g. $\pi-\pi$ stacking between the aromatic compounds (Tavagnacco, Letizia et al., 2011) dipole-dipole interaction between the molecules, electrostatic interactions between the oppositely charged species, etc. Examples of $\pi-\pi$ stacking and electrostatic interaction analysed by the students are shown in Figure 03 and Figure 04. Most of all the participating students learnt to keep patience while doing the MD simulations, as these calculations take from a few minutes to a whole day to complete depending upon the configuration of the machine.

To our delight, we could see these students working enthusiastically, spending longer times in understanding more the structures of each molecule and of the molecular clusters. Later when participants discussed about this workshop among their classmates, we got many new requests from the other students who were not the part of the workshop initially. We allowed the new students to join the workshop who approached us within the 1st week of the workshop but many more students who approached us later, we couldn't took as we had limited resources and the initial phase of the workshop was over. The other participants helped the new joiners while writing the input files. Once the students started getting conclusive results, they were very determined in completing the given task and learning new techniques in the field of molecular simulations. The experience has changed the way now these students learn chemistry and physics of the engineering systems that they encounter. Here is the feedback which we got from the one of the first year undergraduate students, who had attended molecular dynamics workshop.

"Molecular Dynamics Workshop was a great experience. It introduced me to an entire new world of chemistrydescribing how molecules look, how they interact with each other, etc. The workshop helped me by easing the process of understanding chemistry as it is and not as it is in the books. In lectures, we are asked to imagine how molecules react (however imagination is limited), but using Chimera we can build molecules as we want and of any complexity. We get a 3-D overview of how the molecule is. Also, simulating the systems was really fun. Simulating the molecules helps in knowing the behavior of these molecules with each other. It can help us to predict how molecules will behave in different varying environments. We were taught how to analyze a given result of the simulation. We analyzed the results by finding possible interactions between atoms, looking at the molecule's affinity for different solvents when mixed together, checking for the variation of density with temperature and lots more. This workshop has also helped in building team-working skills as we all worked on different parts of the project as a team. The workshop was valuable and was worth dedicating my summer vacation. I hope to learn more regarding molecular dynamics and attend similar other workshops. It is a good idea of learning something beyond the lectures!!!"

Another undergraduate student of chemical engineering wrote:

"A few months ago, I got an opportunity to work on a project involving Molecular dynamics and Simulations. After an introductory workshop that taught us how to use the software, we were each given a system to work on -for the extraction of metal ions from nuclear waste. But what I learnt along the way was much more than that. Essentially, it allowed us to study complex phenomena by the visualization of the structures, interactions and dynamics of the atoms and molecules. Further, by sequentially displaying a series of static frames, of the different states of the same molecule, I was able to create an animation that helped us see the behavior of atoms over time. Although under supervision, I was given the freedom to experiment with the software, "mess around" with the models, and try many "what-ifs" to see what would happen. This process of trial and error helped us learn more, than by simply following the rules. By interacting with the simulations ourselves, through the adjustment of various parameters and inputs, and by watching the results, I was able to construct our own mental pictures about the important 
physical and chemical concepts embodied in the simulations. I was able to understand certain aspects of chemistry, that no textbook or teacher could ever explain. After all, it is said a picture speaks a thousand words. Consequently, a movie is worth a 1000 pictures, and a model is worth a 1000 movies. Naturally, then, a model provides a much larger intellectual space for learners to explore. The experience was truly enriching and profound lessons were learnt, beyond those of the ordinary classroom."

\section{Outcome of the activity}

This activity also encouraged research in the field of molecular modeling at undergraduate level. After finishing the one month of the workshop, almost all the students kept on working on their own beyond the workshop and completed a set of projects of their interests in spare time along with their regular lectures and practical laboratories in just last three months. Their interest in continuing with the project shows their increased interest in the basic science. The quality of work done by these students is good enough to publish papers in international journals. Some of the students also presented a poster on MD simulation in technical fest and won prizes too. Beyond that many of the students further want to take on new projects in the field of molecular dynamics on the topics of their interest.

Similar types of workshops could be conducted in other engineering institutes to encourage students for better understanding and learning of basic chemistry. We have already conducted similar type of workshop in Dr Babasaheb Ambedkar Technological University, Lonere in addition to this workshop and got positive response from students there. Other institutes have also requested us to conduct the similar workshop at their campus. We have developed a MD group which includes group of students participated in the workshop and then we are planning to conduct the similar workshop every year to include fresh students in the group and students participated in previous workshop can help during the training of fresh students. In this manner we can continue the chain every year, even after senior students from the group leave because till then new students will be in the MD group to help in the training of fresh batch every year.

\section{Conclusions}

Molecular dynamic simulation has been implemented for the first time as a teaching tool for undergraduate students in the Institute. This workshop also enabled them to understand chemistry better by relating their theoretical knowledge of chemistry to bulk experimental properties. The ability of modern software to rapidly convert numerical data into understandable three-dimensional representations cannot be ignored. Molecular modeling and simulation is clearly becoming a valuable research tool, which must be included at the undergraduate level.

\section{Acknowledgement}

We would like thank the Information Processing Center (IPC) and Professor M. M. Sharma Library at the Institute of Chemical Technology for providing the computational facility for the workshop. We would also like to thank all the staff members at both the facility for being patient with the students working at odd hours and always being helpful.

\section{References}

Paselk, R. A.; Zoellner, R. W. (2002) Molecular Modeling and Computational Chemistry a Humboldt State University, J. Chem. Educ., 79 (10), 1192.

Whisnant, D. M.; Lever, L. S.; Howe, J. J. (2000) C12O4 in the Stratosphere: A Collaborative Chemistry Project, J. Chem. Educ., 77 (12), 1648.

A.T.S. Taylor and S.E. Feller (2002) Structural Studies of Phycobiliproteins from Spirulina: Combining Spectroscopy, Thermodynamics, and Molecular Modeling in an Undergraduate Biochemistry Experiment", J. Chem. Educ., 79, 1467.

Cramer, C. J.; Kormos, B. L.; Winget, P.; Audette, V. M.; Beebe, J. M.; Brauer, C. S.; Burdick, W. R.; Cochran, E. W.; Eklov, B. M.; Giese, T. J.; Jun, Y.; Kesavan, L. S. D.; Kinsinger, C. R.; Minyaev, M. E.; Rajamani, R.; Salsbury, J. S.; Stubbs, J. M.; Surek, J. T.; Thompson, J. D.; Voelz, V. A.; Wick, C. D.; Zhang, L. (2001) Cooperative Molecular Modeling Exercise--The Hypersurface as Classroom" J. Chem. Educ., 78, 1202.

Berendsen, H. J. C. , van der Spoel, D. \& van Drunen (1995) R. GROMACS: A message-passing parallel molecular dynamics implementation, Comp. Phys. Comm. 91, 43 -56 E Lindahl, B Hess, D Van Der Spoel (2001) GROMACS 3.0: a package for molecular simulation and trajectory analysis, Journal of Molecular Modeling 7 (8), 306-317

H. J. C. Berendsen, J. P. M. Postma, W. F. V. Gusteren, A. Dinola, and J. R. Haak (1984) Molecular dynamics with coupling to an external bath, J. Phys. Chem., 81, 3684.

Pettersen EF, Goddard TD, Huang CC, Couch GS, Greenblatt DM, Meng EC,Ferrin TE. (2004) UCSF Chimera--a visualization system for exploratory research and analysis, J Comput Chem., 13, 1605-12.

Humphrey, W., Dalke, A. and Schulten, K., "VMD - Visual Molecular Dynamics", J. Molec. Graphics, 1996, vol. 14, pp. 33-38

Larry Dukerich (2015) Applying Modeling Instruction to High School Chemistry to Improve Students' Conceptual Understanding, J. Chem. Educ., 92, 1315-1319

Melanie M. Cooper, Leah C. Williams, and Sonia M. Underwood (2015) Student Understanding of Intermolecular Forces: A Multimodal Study, J. Chem. Educ., 92, 1288-1298.

Pallant, A.; Tinker, R. (2004) Reasoning with atomic-scale molecular dynamic models, J. Sci. Educ. Tech., 13, 51-66.

Sabelli, N. H. (1994). On Using Technology for Understanding Science. Interactive Learning Environments, 4, 195-198.

Flöck, D., Rossetti, G., Daidone, I., Amadei, A. and Nola, A. D. (2006), Aggregation of small peptides studied by molecular dynamics simulations, Proteins Structure, Function, and Bioinformatics, 65: 914-921.

M. B. Singh, V. H. Dalvi and V. G. Gaikar (2015) Investigations of clustering of ions and diffusivity in concentrated aqueous solutions of lithium chloride by 
molecular dynamic simulations, RSC Adv., 5, 1532815337.

Tavagnacco, L., Schnupf, U., Mason, P. E., Saboungi, M.L., Cesàro, A., \& Brady, J. W. (2011). Molecular Dynamics Simulation Studies of Caffeine Aggregation in Aqueous Solution. The Journal of Physical Chemistry. B,115(37), 10957-10966. 